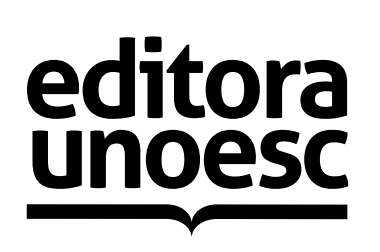

ISSM on-line 2236-6059

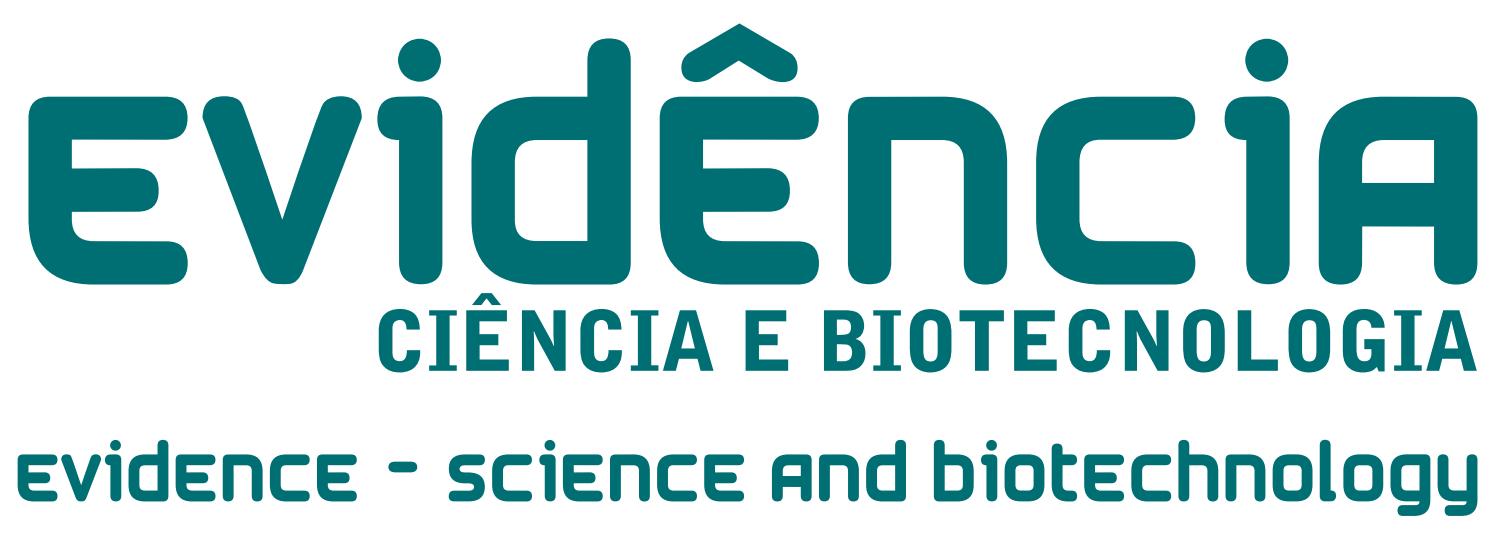

v. 19 n. 1 jan./jun. 2019

DOI desta edição: http://dx.doi.org/10.18593/eba.v19i1 
(C)2019 Editora Unoesc

Direitos desta edição reservados à Editora Unoesc

É proibida a reprodução desta obra, de toda ou em parte, sob quaisquer formas ou por quaisquer meios, sem a permissão expressa da editora.

Dados Internacionais de Catalogação-na-Publicação (CIP)

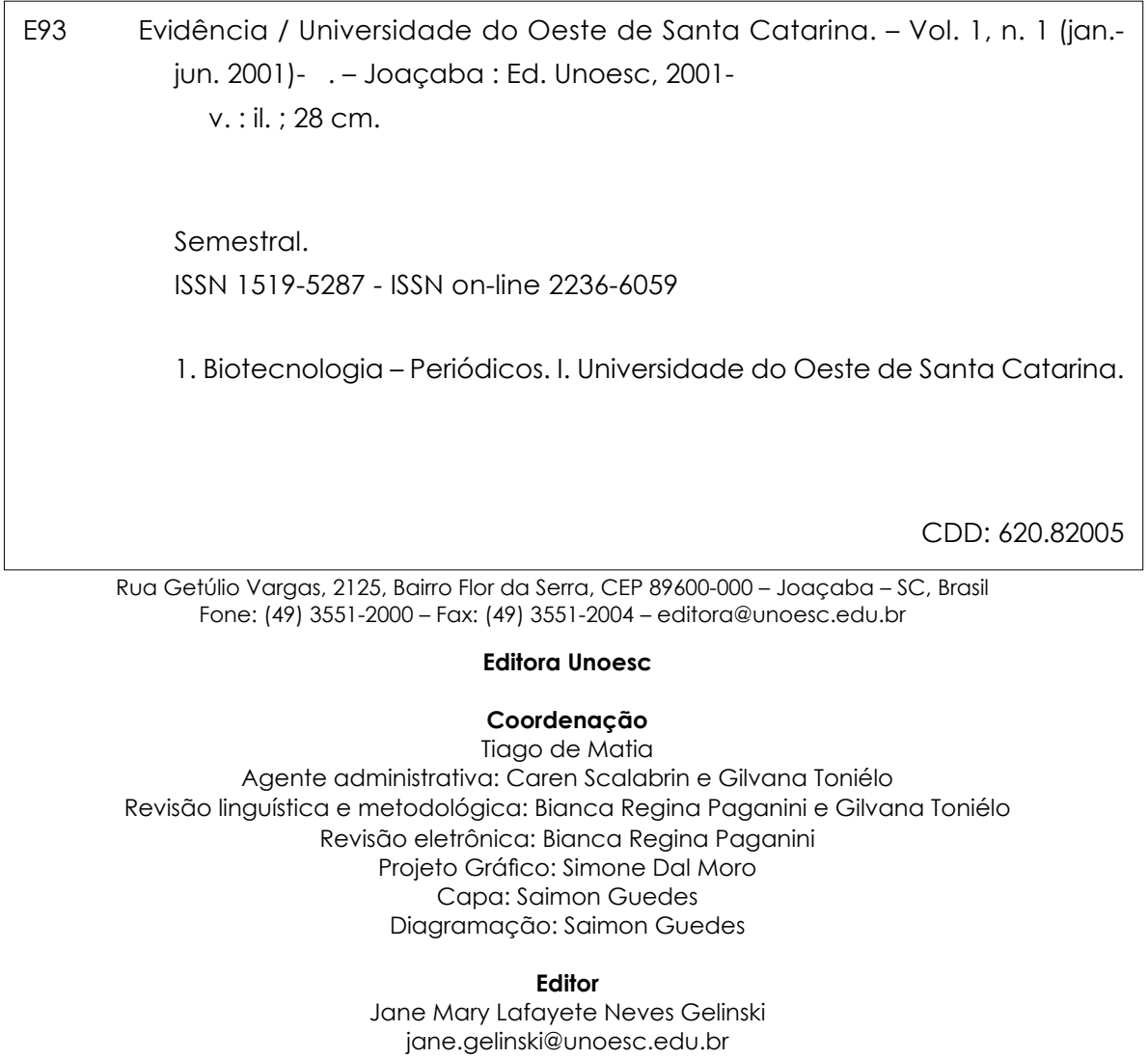

\section{Comissão Editorial}

Dr. Alexis Trott - Universidade do Oeste de Santa Catarina, Brasil Dra. Andréia Antoniuk Presta, Universidade do Oeste de Santa Catarina, Brasil Dr. António André Chivanga Barros - Universidade Regional de Blumenau, Brasil

Dr. César Milton Baratto - Universidade do Oeste de Santa Catarina, Brasil Dra. Eduarda Magalhães Dias Frinhani - Universidade do Oeste de Santa Catarina, Brasil Dra. Estela de Oliveira Nunes - Universidade do Oeste de Santa Catarina, Brasil

Dr. Eugênio Simão - Universidade Federal de Santa Catarina, Brasil Dr. Fabiano Cleber Bertoldi - Epagri, Brasil

Dra. Izildinha Moreno - Instituto de Tecnologia de Alimentos - SP Dra. Jaime Finguerut - Centro de Tecnologia Canavieira

Dra. Jane Lafayette Neves Gelinski - Universidade do Oeste de Santa Catarina, Brasil

Dra. Janice Isabel Druzian - Universidade Federal da Bahia, Brasil

Dr. Jorge Horii - Esalq - Universidade de São Paulo

Dra. Luiz Carlos Basso - Esala - Universidade de São Paulo

Dr. Luiz Severo Silva Júnior - Universidade Estadual de Feira de Santana, Brasil

Dr. Marcelo Fossa da Paz - Universidade Federal da Grande Dourados, Brasil PhD. Marco Antonio Dalbó - Epagri, Brasil

Dra. Maria Ligia Coutinho Carvalhal - Universidade de São Paulo, Brasil

Dr. Paulo Celso de Mello-Farias - Universidade Federal do Rio Grande, Brasil

Dr. Paulo Cesar Poeta Fermino Junior - Universidade Federal do Acre, Brasil

Indexação:

Directory of Open Access Journals: DOAJ

Cite Factor

Academic Journals Database

Sistema Regional de Información en Línea para Revistas Científicas de América Latina, el Caribe, España y Portugal (Latindex) - México

Hemeroteca Latinoamericana (Hela) - México

Índice de Revistas Latinoamericanas en Ciencias (Periódica) - México

SibiNet - Biblioteca Virtual da USP

Sumários.org - Sumários de Revistas Brasileiras

Indexação Compartilhada de Artigos de Periódicos (ICAP)

Biblioteca da Justiça Federal

Diadorim

Este periódico obedece ao procedimento de avaliação de originais double blind review. 


\section{COMTEÚDO}

EDITORIAL

COOKIE ADICIONADO DE FARINHA DE RESÍDUOS DE GUAVIRA:

COMPOSIÇÃO FÍSICO-QUÍMICA E ANÁLISE SENSORIAL

Medino IC, Tonini IGO, Amaral LA, Loubet Filho PS, Santos EF, Novello D

ELABORAÇÃO DE SORVETE DE CUPUAÇU UTILIZANDO FIBRA DE

CASCA DE MARACUJÁ COMO SUBSTITUTO DE GORDURA

Carlos SAV, Amaral LA, Santos MMR, Santee CM, Zampieri DF, Soares WRG, Novello D, Santos EF

CONCENTRAÇÕES DE CITOCININA INFLUENCIAM A MULTIPLICAÇÃO

IN VITRO DE KIWIZEIRO

Arruda AL, Buss M, Nerbass FR, Rufato L

IDENTIFICAÇÃO E AVALIAÇÃO DA SUSCEPTIBILIDADE A

ANTIMICROBIANOS DE SOROTIPOS DE SALMONELLA SP. DE UMA

CADEIA PRODUTIVA DE FRANGOS DE CORTE DO SUL DO BRASIL....

Ansiliero R, Gelinski JMLN, Scheffmacher MGC

ENCEFALOCELE OCCIPITAL: REVISÃO BIBLIOGRÁFICA COM UM

RELATO DE CASO

Mozzer EB, Bonatto KZB, Debiasi MM, D'Agostini FM 
